\title{
ON THE EXISTENCE AND UNIQUENESS OF SOLUTIONS FOR STRONGLY NONLINEAR ELLIPTIC VARIATIONAL PROBLEMS
}

\author{
VESA MUSTONEN* and CHRISTIAN G. SIMADER
}

\section{Introduction}

In this note we are concerned with the existence and uniqueness of solutions of nonlinear variational equations and inequalities for quasilinear elliptic partial differential operators of the form

$$
A u(x)=\sum_{|\alpha| \leqq m}(-1)^{|\alpha|} D^{\alpha}\left(g_{\alpha}\left(D^{\alpha} u(x)\right)\right), \quad x \in \Omega
$$

which can be thought of as a simple nonlinear generalisation of the Laplace operator. Here $\Omega$ is an open bounded subset in $\boldsymbol{R}^{N}$ and the coefficients $g_{\alpha}$ are continuous functions obeying no growth restrictions from above.

The problems of this type have been extensively studied of late in the context of the theory of mappings of monotone type acting between Banach spaces which are determined by the behaviour of the coefficients. When the growth is at most of polynomial type, it is customary to work with reflexive Sobolev spaces (see [2], [4], [15], [16]), but when no such restriction is imposed, it is natural to tackle this problem in the framework of complementary systems of Sobolev-Orlicz spaces (see [6], [7], [8], [9], [10]). In this method the function space, where the problem is to be studied, is determined by the maximal growth of the coefficients. However, the abstract surjectivity results are available for the existence of solutions for the problem, provided an additional coercivity condition is fulfilled. That condition means a restriction for the growth from below. There have been several ways to widen the space between these two growth limitations. One may consider the sums of differential operators such that the principal part is coercive, obeying a polynomial growth condition, while the other terms may have liberal growth (see [3], [5], [11], [18], [19], [20], [21], [22]). The introduction of an anisotropic product of Sobolev spaces allows different growth for various coefficients of the same order ([7]), [10]).

* Supported by an Alexander von Humboldt Foundation Fellowship at the University of Bayreuth while on leave from the University of Oulu.

doi:10.5186/aasfm.1981.0612 
The growth limit from above for the lower order terms can be slightly lifted by the use of the full force of the Sobolev embedding theorem.

The purpose of the present note is to introduce a new rather simple regularisation method which allows more liberty for the coefficients $g_{\alpha}$ of the operator (1.1) than the methods described above and which can be applied to variational inequalities as well as to various boundary value problems. We shall make the operator coercive in some function space by imposing on the functions $g_{\alpha}$ a weak growth restriction from below which only means that $g_{\alpha}(t) \rightarrow \infty$ as $t \rightarrow \infty$ and $g_{\alpha}(t) \rightarrow-\infty$ as $t \rightarrow-\infty$. No growth limitations will be needed from above. To be more precise we introduce our conditions for the coefficients $g_{\alpha}$.

$\left(\mathrm{H}_{1}\right)$ For each $\alpha,|\alpha| \leqq m$, the function $g_{\alpha}: \boldsymbol{R} \rightarrow \boldsymbol{R}$ is continuous. There exists an Orlicz function $M$ and a constant $c_{1}>0$ such that for each $|\alpha| \leqq m$,

$$
g_{\alpha}(t) t \geqq M(t)-c_{1} \text { for all } t \in \boldsymbol{R} \text {. }
$$

$\left(\mathrm{H}_{2}\right)$ For each $\alpha$ with $|\alpha|=m$ the function $g_{\alpha}$ is strictly increasing.

$\left(\mathrm{H}_{3}\right)$ For each $\alpha$ with $|\alpha| \leqq m-1$ the function $g_{\alpha}$ has a representation $g_{\alpha}=\varphi_{\alpha}+r_{\alpha}$, where $r_{\alpha}$ is continuous and bounded and $\varphi_{\alpha}$ is continuous, satisfying the conditions $\varphi_{\alpha}(t) t \geqq 0$ for all $t \in \boldsymbol{R}$ and

$$
\varphi_{\alpha}(t) s \leqq \varphi_{\alpha}(s) s+K_{1} \varphi_{\alpha}(t) t+K_{2}
$$

for all $s, t \in \boldsymbol{R}, K_{1}$ and $K_{2}$ being some positive constants.

We would like to mention that (1.2) guarantees the existence of the representation in $\left(\mathrm{H}_{3}\right)$ for all $|\alpha| \leqq m-1$. On the other hand, it will be possible to relax (1.2) for $|\alpha| \leqq m-1$ provided Poincare's inequality holds in the Sobolev space involved. The inequality (1.3) is well known by the papers dealing with strongly nonlinear elliptic problems also in a more restrictive form (cf. [5], [18], [19], [20], [21]). It obviously holds for all nondecreasing functions (see [20] p. 11). Note also that we have made no oddness assumption for $g_{\alpha}$ (cf. [9], [10]).

Our existence theorems for variational equations and inequalities for the operator (1.1) satisfying the assumptions $\left(\mathrm{H}_{1}\right)$ to $\left(\mathrm{H}_{3}\right)$ will be proved in Section 3. For technical reasons we shall first treat the case where the function $M$ appearing in $\left(\mathrm{H}_{1}\right)$ has a special form $M(t)=c_{0}|t|^{p}$ for some constants $c_{0}>0$ and $1<p<\infty$. The general case will then be derived from this particular case. In Section 4 we give some examples of applications which can be handled by our existence theorems. The last section of this paper is devoted to studying the uniqueness of the solutions of the problems treated in Section 3 when all coefficients $g_{\alpha}$ are strictly increasing. A difficulty arises from the fact that $g_{\alpha}\left(D^{\alpha} u\right)$ for each $|\alpha| \leqq m$ lie in $L^{1}(\Omega)$, only. Therefore, for the uniqueness of the solutions of various interesting problems we shall assume the domain $\Omega$ to be starlike. 
We finally mention that some similar existence results for the Dirichlet boundary value problem have been obtained by R. Landes ([13], [14]) by the Galerkin method. Our study can be easily extended for the more general operators

$$
A u(x)=\sum_{|\alpha| \leqq m}(-1)^{|\alpha|} D^{\alpha} A_{\alpha}\left(x, u(x), D u(x), \ldots, D^{m} u(x)\right), \quad x \in \Omega
$$

by modification of the conditions $\left(\mathrm{H}_{1}\right)$ to $\left(\mathrm{H}_{3}\right)$ for the coefficients $A_{\alpha}$ accordingly.

\section{Preliminaries}

Let $\Omega$ be a bounded domain in $\boldsymbol{R}^{N}$ with $N \geqq 2$, let $m \geqq 1$ be an integer and let $1 \leqq p<\infty$. $W^{m, p}(\Omega)$ will stand for the Sobolev space (of equivalence classes) of functions $u$ defined on $\Omega$ whose distributional derivatives up to the order $m$ lie in $L^{p}(\Omega)$. The norm on $W^{m, p}(\Omega)$ is given by

$$
\|u\|_{m, p}=\left\{\sum_{|\alpha| \leqq m}\left\|D^{\alpha} u\right\|_{L^{p}}^{p}\right\}^{1 / p},
$$

where the summation extends over all multi-indices $\alpha=\left(\alpha_{1}, \ldots, \alpha_{N}\right)$ of non-negative integers with $|\alpha|=\alpha_{1}+\alpha_{2}+\ldots+\alpha_{N} \leqq m$ and $D^{\alpha}=\prod_{i=1}^{N}\left(\partial / \partial x_{i}\right)^{\alpha_{i}} . W_{0}^{m, p}(\Omega)$ will denote the closure in $W^{m, p}(\Omega)$ of $C_{0}^{\infty}(\Omega)$, the infinitely differentiable functions with compact support in $\Omega$. In $W_{0}^{m, p}(\Omega)$ the expression

$$
|u|_{m, p}=\left\{\sum_{\alpha=m}\left\|D^{\alpha} u\right\|_{L^{p}}^{p}\right\}^{1 / p}
$$

defines also a norm which is equivalent to (2.1).

An Orlicz function is any continuous map $M: R \rightarrow R$ which is even, convex and satisfies

$$
\lim _{t \rightarrow 0} \frac{M(t)}{t}=0, \quad \lim _{t \rightarrow \infty} \frac{M(t)}{t}=\infty .
$$

The complementary Orlicz function of $M$ will be denoted by $\bar{M}$. The Orlicz class $K_{M}(\Omega)$ is defined as the set of measurable functions $u$ such that

$$
\int_{\Omega} M(u(x)) d x<\infty
$$

and the Orlicz space $L_{M}(\Omega)$ as the linear hull of $K_{M}(\Omega)$ furnished with the Luxemburg norm

$$
\|u\|_{M}=\inf \left\{\lambda: \int_{\Omega} M\left(\frac{u(x)}{\lambda}\right) d x \leqq 1\right\} .
$$

The closure in $L_{M}(\Omega)$ of bounded functions with compact support in $\Omega$ is denoted by $E_{M}(\Omega)$. The Sobolev-Orlicz space of functions $u$ such that $u$ and its distributional 
derivatives up to the order $m$ lie in $L_{M}(\Omega)$ is denoted by $W^{m} L_{M}(\Omega)$. It becomes a Banach space with the norm

$$
\|u\|_{m, M}=\max \left\{\left\|D^{\alpha} u\right\|_{M}:|\alpha| \leqq m\right\} .
$$

A similar definition applies to the space $W^{m} E_{M}(\Omega)$. For a more detailed discussion on Orlicz spaces we refer to [12].

Let $V$ be a closed subspace of $W^{m, p}(\Omega)$ containing $W_{0}^{m, p}(\Omega)$ and let $s>\max \{N, p\}$. Then the set $W=W^{m+1, s}(\Omega) \cap V$ is a closed subspace of $W^{m+1, s}(\Omega)$. If the domain $\Omega$ has the cone property, it follows from the Sobolev embedding theorem ([1] p. 97, for example) that $W^{m+1, s}(\Omega)$ is continuously embedded in $C_{B}^{m}(\Omega)$, a Banach space under the norm

$$
\|u\|_{m, \infty}=\max \left\{\left\|D^{\alpha} u\right\|_{L^{\infty}}:|\alpha| \leqq m\right\} .
$$

For any $v \in W$ we introduce a partial differential operator

$$
B v(x)=\sum_{|\alpha| \leqq m+1}(-1)^{|\alpha|} D^{\alpha}\left(\left|D^{\alpha} v(x)\right|^{s-2} D^{\alpha} v(x)\right),
$$

which gives rise to a mapping $S$ from $W$ to $W^{*}$ (a duality map with respect to the gauge $\left.\Phi(r)=r^{s-1}\right)$ by the rule

$$
(S(u), v)=\int_{\Omega} \sum_{|\alpha| \leqq m+1}\left|D^{\alpha} u\right|^{s-2} D^{\alpha} u D^{\alpha} v d x,
$$

where $u, v \in W$ and $(.,$.$) denotes the pairing between W$ and $W^{*}$, the dual space of $W$. The map $S$ is monotone, i.e. $(S(u)-S(v), u-v) \geqq 0$ for all $u, v \in W$ and it takes bounded sets of $W$ onto bounded sets of $W^{*}$; indeed,

$$
|(S(u), v)| \leqq\|u\|_{W}^{s-1}\|v\|_{W} \quad \text { for all } u, v \in W .
$$

Moreover, since $(S(u), u)=\|u\|_{W}^{s}, S$ is coercive in $W$, i.e. $(S(u), u)\|u\|_{W}^{-1} \rightarrow \infty$ as $\|u\|_{W} \rightarrow \infty$. Observe finally that $S$ is also continuous and pseudo-monotone (cf. [4]), that is, (PM) whenever $\left(u_{j}\right)$ is a sequence in $W$ which converges weakly to an element $u$ in $W$ (we write $\left.u_{j}-u\right)$ and $\lim \sup \left(S\left(u_{j}\right), u_{j}-u\right) \leqq 0$, it follows that $S\left(u_{j}\right) \rightarrow S(u)$ and $\left(S\left(u_{j}\right), u_{j}\right) \rightarrow(S(u), u)$.

Let us assume that the functions $g_{\alpha}$ satisfy the conditions $\left(\mathrm{H}_{1}\right)$ to $\left(\mathrm{H}_{3}\right)$ introduced in Section 1 for all $|\alpha| \leqq m$. We shall denote

$$
V_{1}=\left\{v \in V: g_{\alpha}\left(D^{\alpha} v\right) \in L^{1}(\Omega) \text { and } g_{\alpha}\left(D^{\alpha} v\right) D^{\alpha} v \in L^{1}(\Omega) \text { for all }|\alpha| \leqq m\right\} .
$$

It is clear that $W \subset V_{1} \subset V$. The semilinear form

$$
a(u, v)=\int_{\Omega} \sum_{|\alpha| \leqq m} g_{\alpha}\left(D^{\alpha} u\right) D^{\alpha} v d x
$$

is well-defined and bounded for all $u \in V_{1}$ and $v \in W$. Therefore we can define a (nonlinear) mapping $T$ from $V_{1}$ to $W^{*}$ by

$$
(T(u), v)=a(u, v) \text { for all } u \in V_{1}, \quad v \in W .
$$


We shall close this section by the following result which is an immediate consequence of the theory of mappings of monotone type acting between reflexive Banach spaces.

Lemma 1. Let $\Omega$ be a bounded domain in $\boldsymbol{R}^{N}$ having the cone property, let $K_{1}$ be a closed convex subset of $W$ containing the origin, let the functions $g_{\alpha}$ satisfy the conditions $\left(\mathrm{H}_{1}\right)$ to $\left(\mathrm{H}_{3}\right)$ and let the mappings $S$ and $T$ be defined by (2.4) and (2.6), respectively. Then for any $f \in W^{*}$ and any $\sigma>0$ the variational inequality

$$
\sigma(S(u), v-u)+(T(u), v-u) \geqq(f, v-u) \text { for all } v \in K_{1}
$$

admits a solution $u$ in $K_{1}$.

Proof. It suffices to verify that the mapping $\sigma S+T$ from $W$ to $W^{*}$ is continuous, pseudo-monotone and coercive (see [16] p. 247, for example). The continuity and pseudo-monotonicity of $S$ are already clear and the respective properties for $\sigma S+T$ follow from the Sobolev embedding theorem (cf. [4]). By $\left(\mathrm{H}_{1}\right),(T(v), v) \geqq$ $-c_{1}$ for all $v \in W$, implying that

$$
\sigma(S(v), v)+(T(v), v)\|v\|_{W^{-1}} \geqq \sigma\|v\|_{W}^{S^{-1}}-c_{1}\|v\|_{W}^{-1} .
$$

Thus $\sigma S+T$ is also coercive.

\section{Existence theorems}

We shall apply Lemma 1 to produce existence theorems for variational inequalities and boundary value problems for the elliptic operator (1.1). To reduce the use of the rather complicated Sobolev-Orlicz spaces to the minimum we shall first deal with the particular case where the function $M$ appearing in the condition $\left(\mathrm{H}_{1}\right)$ has the shape

$$
M(t)=c_{0}|t|^{p}
$$

for some $p$ with $1<p<\infty$ and $c_{0}>0$. This condition,

$\left(\mathrm{H}_{1}\right)^{*}$ For each $\alpha,|\alpha| \leqq m$, the function $g_{\alpha}: \boldsymbol{R} \rightarrow \boldsymbol{R}$ is continuous. There are constants $c_{0}, c_{1}>0$ and $p$ with $1<p<\infty$ such that for each $|\alpha| \leqq m$,

$$
g_{\alpha}(t) t \geqq c_{0}|t|^{p}-c_{1} \text { for all } t \in \boldsymbol{R}
$$

enables us to stay within the frames of reflexive Sobolev spaces. The general case with the condition $\left(\mathrm{H}_{1}\right)$ we shall treat by making the necessary changes in the proof.

Let $V$ be a closed subspace of $W^{m, p}(\Omega)$ containing $W_{0}^{m, p}(\Omega)$, where $W^{m, p}(\Omega)$ is determined through the condition $\left(\mathrm{H}_{1}\right)^{*}$. By choosing $s>\max \{N, p\}$ we define $W=W^{m+1, s}(\Omega) \cap V$. Then we have 
Theorem 1. Let $\Omega$ be a bounded domain in $\boldsymbol{R}^{N}$ having the cone property, let the functions $g_{\alpha}$ satisfy the conditions $\left(\mathrm{H}_{1}\right)^{*},\left(\mathrm{H}_{2}\right)$ and $\left(\mathrm{H}_{3}\right)$, and let $K$ be a closed convex subset of $V$ containing the origin. Then for each $f \in V^{*}$ the variational inequality

$$
a(u, w-u) \geqq(f, w-u) \text { for all } w \in W \cap K
$$

admits a solution $u$ in $K \cap V_{1}$.

Proof of Theorem 1. To invoke Lemma 1 we make two observations. Firstly, the natural injection map $i$ of $W$ to $V$ is continuous. Thus $V^{*} \subset W^{*}$. For any $f \in V^{*}$ we shall write $f \in W^{*}$ instead of the more precise statement $i^{*} f \in W^{*}$. Secondly, when $K$ is a closed convex set in $V$ the set $K_{1}=K \cap W$ inherits the same property as a subset of $W$. Consequently, by Lemma 1 , for any $n \in N$ there exists $u_{n} \in K_{1}$ such that

$$
\frac{1}{n}\left(S\left(u_{n}\right), v-u_{n}\right)+\left(T\left(u_{n}\right), v-u_{n}\right) \geqq\left(f, v-u_{n}\right)
$$

for all $v \in K_{1}$. Setting $v=0 \in K_{1}$ and bearing in mind (2.5) and (2.6) we get

$$
\frac{1}{n}\left(S\left(u_{n}\right), u_{n}\right)+\int_{\Omega} \sum_{|\alpha| \leqq m} g_{\alpha}\left(D^{\alpha} u_{n}\right) D^{\alpha} u_{n} d x \leqq\left(f, u_{n}\right)
$$

for all $n \in N$. By $(2.4)$ and $\left(\mathrm{H}_{1}\right)^{*}$ we have further

$$
\frac{1}{n}\left\|u_{n}\right\|_{W}^{s}+c_{0}\left\|u_{n}\right\|_{V}^{p}-c_{1} \leqq c_{2}\left\|u_{n}\right\|_{V}
$$

with $c_{2}$ being some positive constant. Hence we can conclude that the real-valued sequences $\left\{\left\|u_{n}\right\|_{V}\right\}, \quad\left\{(1 / n)\left\|u_{n}\right\|_{W}^{s}\right\}$ and $\left\{\int_{\Omega} \sum_{|\alpha| \leqq m} g_{\alpha}\left(D^{\alpha} u_{n}\right) D^{\alpha} u_{n} d x\right\}$ remain uniformly bounded. Since $V$ is a reflexive Banach space, there exists a subsequence of $\left\{u_{n}\right\}$ such that (we do not change the notation, for simplicity) $u_{n} \rightarrow u$. As $K$ is also weakly closed, $u \in K$. We shall show that $u$ is a solution of (3.1). For showing this it is sufficient to establish the following four assertions for some further subsequence of $\left\{u_{n}\right\}$.

$$
\begin{gathered}
\lim \sup \left\{\frac{1}{n}\left(S\left(u_{n}\right), w-u_{n}\right)\right\} \leqq 0 \quad \text { for any } \quad w \in W, \\
\int_{\Omega} g_{\alpha}\left(D^{\alpha} u\right) D^{\alpha} u d x \leqq \liminf \int_{\Omega} g_{\alpha}\left(D^{\alpha} u_{n}\right) D^{\alpha} u_{n} d x \quad \text { for all }|\alpha| \leqq m, \\
\lim \int_{\Omega} g_{\alpha}\left(D^{\alpha} u_{n}\right) D^{\alpha} w d x=\int_{\Omega} g_{\alpha}\left(D^{\alpha} u\right) D^{\alpha} w d x \text { for all } w \in W, \\
u \in V_{1} .
\end{gathered}
$$


Indeed, provided that (1) - (4) has been verified, we obtain from (3.2) for any $w=v$ in $W \cap K$,

$$
\begin{gathered}
(f, w-u)=\lim \left(f, w-u_{n}\right) \leqq \lim \sup \left\{\frac{1}{n}\left(S\left(u_{n}\right), w-u_{n}\right)\right\} \\
+\lim \sup \left\{\int_{\Omega} \sum_{|\alpha| \leqq m} g_{\alpha}\left(D^{\alpha} u_{n}\right)\left(D^{\alpha} w-D^{\alpha} u_{n}\right) d x\right\} \\
\leqq \lim \int_{\Omega} \sum_{|\alpha| \leqq m} g_{\alpha}\left(D^{\alpha} u_{n}\right) D^{\alpha} w d x-\sum_{|\alpha| \leqq m}\left\{\lim \inf \int_{\Omega} g_{\alpha}\left(D^{\alpha} u_{n}\right) D^{\alpha} u_{n} d x\right\} \\
\leqq \int_{\Omega} \sum_{|\alpha| \leqq m} g_{\alpha}\left(D^{\alpha} u\right) D^{\alpha} w d x-\int_{\Omega} \sum_{|\alpha| \leqq m} g_{\alpha}\left(D^{\alpha} u\right) D^{\alpha} u d x=a(u ; w-u),
\end{gathered}
$$

which will prove (3.1), $u$ being in $K \cap V_{1}$.

To show (1) we remark that $(1 / n)\left\|u_{n}\right\|_{W}^{s-1} \rightarrow 0$ as $n \rightarrow \infty$, because $\left\{(1 / n)\left\|u_{n}\right\|_{W}^{s}\right\}$ is bounded. Since $\left(S\left(u_{n}\right), u_{n}\right) \geqq 0$, we have

$$
\frac{1}{n}\left(S\left(u_{n}\right), w-u_{n}\right) \leqq \frac{1}{n}\left(S\left(u_{n}\right), w\right) \leqq \frac{1}{n}\left\|u_{n}\right\|_{W}^{s-1}\|w\|_{W},
$$

which implies the assertion (1).

The next step is to show that (2) and (3) are true for all $|\alpha| \leqq m-1$. Indeed, as

$$
\int_{\Omega} \sum_{|\alpha| \leqq m} g_{\alpha}\left(D^{\alpha} u_{n}\right) D^{\alpha} u_{n} d x \leqq c_{3} \text { for all } n \in \mathbf{N}
$$

with $c_{3}$ a positive constant, we have, in view of $\left(\mathrm{H}_{3}\right)$, also

$$
\int_{\Omega} \sum_{|\alpha| \leqq m} \varphi_{\alpha}\left(D^{\alpha} u_{n}\right) D^{\alpha} u_{n} d x \leqq c_{4}, \quad \int_{\Omega} \sum_{|\alpha| \leqq m}\left|g_{\alpha}\left(D^{\alpha} u_{n}\right) D^{\alpha} u_{n}\right| d x \leqq c_{4}
$$

for all $n \in N, c_{4}$ being again some positive constant. On the other hand, by the Sobolev embedding theorem, $D^{\alpha} u_{n}(x) \rightarrow D^{\alpha} u(x)$ a.e. in $\Omega$ for all $|\alpha| \leqq m-1$ for some subsequence. By Fatou's lemma

$$
\int_{\Omega} \varphi_{\alpha}\left(D^{\alpha} u\right) D^{\alpha} u d x \leqq \liminf \int_{\Omega} \varphi_{\alpha}\left(D^{\alpha} u_{n}\right) D^{\alpha} u_{n} d x .
$$

By the dominated convergence theorem $r_{\alpha}\left(D^{\alpha} u_{n}\right) \rightarrow r_{\alpha}\left(D^{\alpha} u\right)$ in $L^{p^{\prime}}(\Omega)$ and hence

$$
\lim \int_{\Omega} r_{\alpha}\left(D^{\alpha} u_{n}\right) D^{\alpha} u_{n} d x=\int_{\Omega} r_{\alpha}\left(D^{\alpha} u\right) D^{\alpha} u d x
$$

for all $|\alpha| \leqq m-1$. Now (3.4) and (3.5) imply that (2) holds true and that also $g_{a}\left(D^{\alpha} u\right) D^{\alpha} u \in L^{1}(\Omega)$ for all $|\alpha| \leqq m-1$.

For any $\delta>0$,

$$
\left|\varphi_{\alpha}\left(D^{\alpha} u_{n}(x)\right)\right| \leqq \max _{|t| \leqq \delta^{-1}}\left|\varphi_{\alpha}(t)\right|+\delta \varphi_{\alpha}\left(D^{\alpha} u_{n}(x)\right) D^{\alpha} u_{n}(x) .
$$

Applying Vitali's convergence theorem we obtain $\varphi_{\alpha}\left(D^{\alpha} u_{n}\right) \rightarrow \varphi_{\alpha}\left(D^{\alpha} u\right)$ and 
$g_{\alpha}\left(D^{\alpha} u_{n}\right) \rightarrow g_{\alpha}\left(D^{\alpha} u\right)$ in $L^{1}(\Omega)$ for all $|\alpha| \leqq m-1$. Finally, since $D^{\alpha} w \in L^{\infty}(\Omega)$ whenever $w \in W$ and $|\alpha| \leqq m$, we obtain (3) for all $|\alpha| \leqq m-1$.

To be able to establish the assertions (2) and (3) also for all $|\alpha|=m$ it is clearly sufficient, by exactly the same argument as above, to show that

$$
D^{\alpha} u_{n}(x) \rightarrow D^{\alpha} u(x) \text { a.e. in } \Omega \text { for all }|\alpha|=m .
$$

Then it will also be plain that $g_{\alpha}\left(D^{\alpha} u\right) D^{\alpha} u \in L^{1}(\Omega)$ and $g_{\alpha}\left(D^{\alpha} u\right) \in L^{1}(\Omega)$ for all $|\alpha| \leqq m$, which means that $u \in V_{1}$, i.e. the assertion (4) holds true, too. Hence the proof of Theorem 1 is completed by verification of the claim (3.6). This we shall do by means of the following three lemmas.

Lemma 2. For each $\alpha$ with $|\alpha|=m$ there exists a function $h_{\alpha}$ in $L^{1}(\Omega)$ such that $g_{\alpha}\left(D^{\alpha} u_{n}\right) \rightarrow h_{\alpha}$ in $L^{1}(\Omega)$ for some subsequence of $\left\{u_{n}\right\}$.

Proof. It is sufficient to show that $\left\{g_{\alpha}\left(D^{\alpha} u_{n}\right)\right\}$ is uniformly equi-integrable for each $|\alpha|=m$. By the conditions $\left(\mathrm{H}_{1}\right)^{*}$ and $\left(\mathrm{H}_{2}\right)$ each $g_{\alpha}$ can be written in the form $g_{\alpha}=\varphi_{\alpha}+c_{\alpha}$, where $\varphi_{\alpha}(t)=g_{\alpha}(t)-g_{\alpha}(0)$ and $c_{\alpha}=g_{\alpha}(0)$. Thus $\varphi_{\alpha}(t) t \geqq 0$ for all $\boldsymbol{t} \in \boldsymbol{R}$. For any $\delta>0$ we have

$$
\varphi_{\alpha}\left(D^{\alpha} u_{n}(x)\right) \leqq \max _{|t| \leqq \delta^{-1}}\left|\varphi_{\alpha}(t)\right|+\delta \varphi_{\alpha}\left(D^{\alpha} u_{n}(x)\right) D^{\alpha} u_{n}(x),
$$

where $\left\{\varphi_{\alpha}\left(D^{\alpha} u_{n}\right) D^{\alpha} u_{n}\right\}$ is uniformly bounded in $L^{1}(\Omega)$ by a constant $c_{4}$ for all $|\alpha|=m$. For any measurable subset $E$ of $\Omega$ we then have

$$
\int_{E}\left|\varphi_{\alpha}\left(D^{\alpha} u_{n}\right)\right| d x \leqq \int_{E} K_{\delta} d x+\delta c_{4}=K_{\delta} \mu(E)+\delta c_{4},
$$

where $K_{\delta}$ is a constant depending on $\delta$. जiven $\varepsilon>0$ let $\delta$ be such that $\delta c_{4}<\varepsilon / 2$ and let $\mu(E)<\varepsilon / 2 K_{\delta}$. Hence we can see that $\left\{\varphi_{\alpha}\left(D^{\alpha} u_{n}\right)\right\}$ and therefore also $\left\{g_{\alpha}\left(D^{\alpha} u_{n}\right)\right\}$ are equi-integrable.

In the following lemma we denote, for convenience, $h_{\alpha}=g_{\alpha}\left(D^{\alpha} u\right)$ for each $\alpha$ with $|\alpha| \equiv m-\mathrm{I}$. Thus we can write $g_{\alpha}\left(D^{\alpha} u_{n}\right) \rightarrow h_{\alpha}$ in $L^{1}(\Omega)$ for all $|\alpha| \leqq m$, where in fact $g_{\alpha}\left(D^{\alpha} u_{n}\right) \rightarrow h_{\alpha}$ in $L^{1}(\Omega)$ for all $|\alpha| \leqq m-1$.

Lemma 3. $\lim \sup \int_{\Omega} \sum_{|\alpha| \leqq m}\left\{g_{\alpha}\left(D^{\alpha} u_{n}\right)-h_{\alpha}\right\} D^{\alpha} u_{n} d x \leqq 0$.

Proof. Since $\left\{u_{n}\right\} \subset K_{1}=K \cap W$, we may substitute $v=u_{j}(1 \leqq j \leqq n)$ in (3.2) to get

and hence

$$
\begin{aligned}
\frac{1}{n}\left(S\left(u_{n}\right), u_{j}-u_{n}\right)+ & \int_{\Omega} \sum_{|\alpha| \leqq m} g_{\alpha}\left(D^{\alpha} u_{n}\right)\left(D^{\alpha} u_{j}-D^{\alpha} u_{n}\right) d x \\
& \geqq\left(f, u_{j}-u_{n}\right)
\end{aligned}
$$

$$
\begin{gathered}
\int_{\Omega} \sum_{|\alpha| \leqq m} g_{\alpha}\left(D^{\alpha} u_{n}\right) D^{\alpha} u_{n} d x \leqq \frac{1}{n}\left(S\left(u_{n}\right), u_{j}-u_{n}\right)+\left(f, u_{n}-u_{j}\right) \\
+\int_{\Omega} \sum_{|\alpha| \leqq m} g_{\alpha}\left(D^{\alpha} u_{n}\right) D^{\alpha} u_{j} d x .
\end{gathered}
$$


As $\left(S\left(u_{n}\right), u_{n}\right) \geqq 0$ we have further

$$
\begin{gathered}
\int_{\Omega} \sum_{|\alpha| \leqq m}\left\{g_{\alpha}\left(D^{\alpha} u_{n}\right)-h_{\alpha}\right\} D^{\alpha} u_{n} d x \leqq \frac{1}{n}\left(S\left(u_{n}\right), u_{j}\right)+\left(f, u_{n}-u_{j}\right) \\
+\int_{\Omega} \sum_{|\alpha| \leqq m} g_{\alpha}\left(D^{\alpha} u_{n}\right) D^{\alpha} u_{j} d x-\int_{\Omega} \sum_{|\alpha| \leqq m} h_{\alpha} D^{\alpha} u_{n} d x
\end{gathered}
$$

Letting $n \rightarrow \infty$ and keeping $j$ fixed we can conclude

$$
\begin{gathered}
\lim \sup _{n} \int_{\Omega} \sum_{|\alpha| \leqq m}\left\{g_{\alpha}\left(D^{\alpha} u_{n}\right)-h_{\alpha}\right\} D^{\alpha} u_{n} d x \leqq\left(f, u-u_{j}\right) \\
\quad+\int_{\Omega} \sum_{|\alpha| \leqq m} h_{\alpha} D^{\alpha} u_{j} d x-\liminf _{n} \int_{\Omega} \sum_{|\alpha| \leqq m} h_{\alpha} D^{\alpha} u_{n} d x .
\end{gathered}
$$

On the other hand, we can deduce from (3.7) also that

$$
\int_{\Omega} \sum_{|\alpha| \leqq m} h_{\alpha} D^{\alpha} u_{j} d x \geqq\left(f, u_{j}-u\right)-\lim \sup _{n} \int_{\Omega} \sum_{|\alpha| \leqq m}\left|g_{\alpha}\left(D^{\alpha} u_{n}\right) D^{\alpha} u_{n}\right| d x
$$

for all $j \in N$, where we already know that $\sum_{|\alpha| \leqq m}\left\|g_{\alpha}\left(D^{\alpha} u_{n}\right) D^{\alpha} u_{n}\right\|_{L^{1}} \leqq c_{4}$ for all $n \in N$. Hence

$$
-\liminf _{n} \int_{\Omega} \sum_{|\alpha| \leqq m} h_{\alpha} D^{\alpha} u_{n} d x \leqq c_{4}
$$

Finally we can select from the sequence $\left\{u_{j}\right\}$ such a subsequence $\left\{u_{j_{k}}\right\}$ that

$$
\lim _{k \rightarrow \infty} \int_{\Omega} \sum_{|\alpha| \leqq m} h_{\alpha} D^{\alpha} u_{j_{k}} d x=\lim \inf _{n} \int_{\Omega} \sum_{|\alpha| \leqq m} h_{\alpha} D^{\alpha} u_{n} d x,
$$

which enables us to conclude the assertion of Lemma 3 from (3.8).

Lemma 4. $D^{\alpha} u_{n}(x) \rightarrow D^{\alpha} u(x)$ a.e. in $\Omega$ for all $|\alpha|=m$ for some subsequence of $\left\{u_{n}\right\}$.

Proof. We denote

$$
q_{n}(x)=\sum_{|\alpha|=m}\left\{g_{\alpha}\left(D^{\alpha} u_{n}(x)\right)-g_{\alpha}\left(D^{\alpha} u(x)\right)\right\}\left(D^{\alpha} u_{n}(x)-D^{\alpha} u(x)\right) .
$$

In view of $\left(\mathrm{H}_{2}\right)$ the convergence of $D^{\alpha} u_{n}(x)$ to $D^{\alpha} u(x)$ almost everywhere for all $|\alpha|=m$ is implied by $q_{n}(x) \rightarrow 0$ a.e. in $\Omega$ (cf. [14], Lemma 6). In fact, as $q_{n}(x) \geqq 0$ for all $x \in \Omega$, it is sufficient to show that

$$
\lim \sup _{n} \int_{\Omega_{k}} q_{n}(x) d x \leqq \varepsilon_{k},
$$

where $\varepsilon_{k} \rightarrow 0$ as $k \rightarrow \infty$ and $\left\{\Omega_{k}\right\}$ is a growing sequence of the subsets of $\Omega$ such that $\mu\left(\Omega \backslash \Omega_{k}\right) \rightarrow 0$ as $k \rightarrow \infty$.

To this end we define for any $k=1,2, \ldots$

$$
\Omega_{k}=\left\{x \in \Omega:\left|D^{\alpha} u(x)\right| \leqq k \text { and }\left|h_{\alpha}(x)\right| \leqq k \text { for all }|\alpha| \leqq m\right\} .
$$


Then we observe that

and

$$
\begin{aligned}
& \lim _{n} \int_{Q_{k}} \sum_{|\alpha|=m} g_{\alpha}\left(D^{\alpha} u\right)\left(D^{\alpha} u_{n}-D^{\alpha} u\right) d x=0, \\
& \lim _{n} \int_{\Omega_{k}} \sum_{|\alpha|=m} h_{\alpha}\left(D^{\alpha} u_{n}-D^{\alpha} u\right) d x=0
\end{aligned}
$$

$$
\lim _{n} \int_{\Omega_{k}} \sum_{|\alpha|=m}\left(h_{\alpha}-g_{\alpha}\left(D^{\alpha} u_{n}\right)\right) D^{\alpha} u d x=0
$$

for any fixed $k$. Moreover, by the inequality (1.3) of $\left(\mathrm{H}_{3}\right)$ we have for all $|\alpha| \leqq$ $m-1$,

$$
\left\{\varphi_{\alpha}\left(D^{\alpha} u\right)-\varphi_{\alpha}\left(D^{\alpha} u_{n}\right)\right\} D^{\alpha} u_{n} \leqq K_{1} \varphi_{\alpha}\left(D^{\alpha} u\right) D^{\alpha} u+K_{2},
$$

where $\varphi_{\alpha}\left(D^{\alpha} u\right) D^{\alpha} u \in L^{1}(\Omega)$. Since $\left\{\varphi_{\alpha}\left(D^{\alpha} u_{n}(x)\right)-\varphi_{\alpha}\left(D^{\alpha} u(x)\right)\right\} D^{\alpha} u_{n}(x) \rightarrow 0$ a.e. in $\Omega$ for all $|\alpha| \leqq m-1$ and since its positive part is dominated by a function in $L^{1}(\Omega)$, we can deduce by the dominated convergence theorem that

Since also

$$
\lim \sup \int_{\Omega}\left\{\varphi_{\alpha}\left(D^{\alpha} u_{n}\right)-\varphi_{\alpha}\left(D^{\alpha} u\right)\right\} D^{\alpha} u_{n} d x \leqq 0 .
$$

$$
\left\{r_{\alpha}\left(D^{\alpha} u\right)-r_{\alpha}\left(D^{\alpha} u_{n}\right)\right\} D^{\alpha} u_{n} \rightarrow 0 \text { in } L^{1}(\Omega) \text { for all }|\alpha| \leqq m-1,
$$

we get (with the convention $h_{\alpha}=g_{\alpha}\left(D^{\alpha} u\right)$ for all $|\alpha| \leqq m-1$ )

$$
\lim \sup \int_{\Omega} \sum_{|\alpha| \leqq m-1}\left\{h_{\alpha}-g_{\alpha}\left(D^{\alpha} u_{n}\right)\right\} D^{\alpha} u_{n} d x \leqq 0 .
$$

As a consequence of (3.10), (3.11), (3.12) and (3.14) we can derive

$$
\begin{gathered}
\limsup \int_{\Omega_{k}} q_{n}(x) d x=\lim \sup \int_{\Omega_{k}} \sum_{|\alpha|=m} g_{\alpha}\left(D^{\alpha} u_{n}\right)\left(D^{\alpha} u_{n}-D^{\alpha} u\right) d x \\
\quad=\lim \sup \int_{\Omega_{k}} \sum_{|\alpha|=m}\left\{g_{\alpha}\left(D^{\alpha} u_{n}\right)-h_{\alpha}\right\} D^{\alpha} u_{n} d x \\
\quad \leqq \limsup \int_{\boldsymbol{\Omega}} \sum_{|\alpha| \leqq m}\left\{g_{\alpha}\left(D^{\alpha} u_{n}\right)-h_{\alpha}\right\} D^{\alpha} u_{n} d x \\
\quad+\limsup \int_{\Omega \backslash \Omega_{k}} \sum_{|\alpha|=m}\left\{h_{\alpha}-g_{\alpha}\left(D^{\alpha} u_{n}\right)\right\} D^{\alpha} u_{n} d x .
\end{gathered}
$$

Taking into account that the functions $g_{\alpha}$ for each $|\alpha|=m$ satisfy the inequality (1.3) of $\left(\mathrm{H}_{3}\right)$, we can apply the argument of [13] p. 20 to show that

$$
\sum_{|\alpha|=m}\left\{h_{\alpha}(x)-g_{\alpha}\left(D^{\alpha} u_{n}(x)\right)\right\} D^{\alpha} u_{n}(x) \leqq \Gamma(x)
$$

for all $n \in N, \Gamma$ being some function in $L^{1}(\Omega)$. From (3.15) and Lemma 3 it then follows that

$$
\lim \sup \int_{\Omega_{k}} q_{n}(x) d x \leqq \int_{\Omega \backslash \Omega_{k}} \Gamma(x) d x
$$


where $\varepsilon_{k}=\int_{\Omega \backslash \Omega_{k}} \Gamma(x) d x \rightarrow 0$ as $k \rightarrow \infty$, which completes the proof of Lemma 4 and also the proof of Theorem 1.

For various boundary value problems for the operator (1.1) subject to the boundary constraints associated to the subspace $V$ with $W_{0}^{m, p}(\Omega) \subset V \subset W^{m, p}(\Omega)$, where $p$ is determined by the condition $\left(\mathrm{H}_{1}\right)^{*}$, we have the following theorem:

Theorem 2. Let $\Omega$ be a bounded domain in $\boldsymbol{R}^{N}$ having the cone property and let the functions $g_{\alpha}$ satisfy the conditions $\left(\mathrm{H}_{1}\right)^{*},\left(\mathrm{H}_{2}\right)$ and $\left(\mathrm{H}_{3}\right)$. Then for each $f \in V^{*}$ there exists $u$ in $V_{1}$ such that

$$
\int_{\Omega} \sum_{|\alpha| \leqq m} g_{\alpha}\left(D^{\alpha} u\right) D^{\alpha} w d x=(f, w) \text { for all } w \in W,
$$

while

$$
\int_{\Omega} \sum_{|\alpha| \leqq m} g_{\alpha}\left(D^{\alpha} u\right) D^{\alpha} u d x \leqq(f, u) .
$$

Proof. We may choose $K=V$. in the proof of Theorem 1. Setting $v=w+u_{n}$ in (3.2) with $w$ arbitrary in $W$ we get

$$
\frac{1}{n}\left(S\left(u_{n}\right), w\right)+\int_{\Omega} \sum_{|\alpha| \leqq m} g_{\alpha}\left(D^{\alpha} u_{n}\right) D^{\alpha} w d x=(f, w) .
$$

Letting here $n \rightarrow \infty$ we obtain (3.16). Furthermore, if we substitute $w=0$ in (3.1), we obtain (3.17).

Remark 1. When the two norms $\|\cdot\|_{m, p}$ and $|\cdot|_{m, p}$ are equivalent in $V$ (i.e. Poincaré's inequality holds), the coercivity condition $(1.2)^{*}$ of $\left(\mathrm{H}_{1}\right)^{*}$ can be relaxed for the functions $g_{\alpha}$ with $|\alpha| \leqq m-1$. Such is the case when $V=W_{0}^{m, p}(\Omega)$, for example.

Next we shall tackle the general case where the conditions $\left(\mathrm{H}_{1}\right),\left(\mathrm{H}_{2}\right)$ and $\left(\mathrm{H}_{3}\right)$ are assumed. Let $V$ be a closed subspace of $W^{m, 1}(\Omega)$ containing $W_{0}^{m, 1}(\Omega)$. Selecting $s>N$ we define $W=W^{m+1, s}(\Omega) \cap V$. Let further $E_{M}(\Omega), W^{m} E_{M}(\Omega)$ and $E_{\bar{M}}(\Omega)$ be the spaces determined through the function $M$ appearing in the condition $\left(\mathrm{H}_{1}\right)$. We note that each $f \in W^{-m} E_{\bar{M}}(\Omega)=\left\{f=\sum_{|\alpha| \leqq m}(-1)^{|\alpha|} D^{\alpha} f_{\alpha}: f_{\alpha} \in E_{\bar{M}}(\Omega)\right.$ for all $\left.|\alpha| \leqq m\right\}$ defines an element in $W^{*}$ by

$$
(f, w)=\int_{\Omega} \sum_{|\alpha| \leqq m} f_{\alpha} D^{\alpha} w d x,
$$

while

$$
|(f, w)| \leqq \text { const. }\|w\|_{W} \sum_{|\alpha| \leqq m}\left\|f_{\alpha}\right\|_{M} .
$$

Now we are in a position to prove

Theorem 3. Let $\Omega$ be a bounded domain in $\boldsymbol{R}^{N}$ having the cone property, let the functions $g_{\alpha}$ satisfy the conditions $\left(\mathrm{H}_{1}\right),\left(\mathrm{H}_{2}\right)$ and $\left(\mathrm{H}_{3}\right)$ and let $K$ be a closed con- 
vex subset of $V$ containing the origin. Then for each $f \in W^{-m} E_{\bar{M}}(\Omega)$ the variational inequality

$$
a(u, w-u) \geqq(f, w-u) \text { for all } w \in K \cap W
$$

admits a solution $u$ in $K \cap V_{1} \cap W^{m} L_{M}(\Omega)$.

Proof. We shall only indicate the changes which are necessary in the run of the proof of Theorem 1.

The inequality (3.2) obviously holds for all $v \in K_{1}=K \cap W$. Setting $w=0$ in (3.2) we get for all $n \in N$,

$$
\frac{1}{n}\left(S\left(u_{n}\right), u_{n}\right)+\int_{\Omega} \sum_{|\alpha| \leqq m} g_{\alpha}\left(D^{\alpha} u_{n}\right) D^{\alpha} u_{n} d x \leqq \int_{\Omega} \sum_{|\alpha| \leqq m} f_{\alpha} D^{\alpha} u_{n} d x .
$$

By $\left(\mathrm{H}_{1}\right)$ and Young's inequality ([12] p. 135) we have further

$$
\begin{gathered}
\frac{1}{n}\left\|u_{n}\right\|_{W}^{s}+\int_{\Omega} \sum_{|\alpha| \leqq m} M\left(D^{\alpha} u_{n}\right) d x-c_{1} \leqq \int_{\Omega} \sum_{|\alpha| \leqq m} \bar{M}\left(2 f_{\alpha}\right)+M\left(\frac{1}{2} D^{\alpha} u_{n}\right) d x \\
\leqq c_{5}+\frac{1}{2} \int_{\Omega} \sum_{|\alpha| \leqq m} M\left(D^{\alpha} u_{n}\right) d x,
\end{gathered}
$$

where we have used the fact that $f_{\alpha} \in E_{\bar{M}}(\Omega)$ for all $|\alpha| \leqq m$ and where $c_{5}>0$ is a constant. As a consequence we can conclude that the real-valued sequences $\left\{(1 / n)\left\|u_{n}\right\|_{W}^{s}\right\},\left\{\sum_{|\alpha| \leqq m} \int_{\Omega} M\left(D^{\alpha} u_{n}\right) d x\right\}$ and $\left\{\sum_{|\alpha| \leqq m} \int_{\Omega} g_{\alpha}\left(D^{\alpha} u_{n}\right) D^{\alpha} u_{n} d x\right\}$ are uniformly bounded. By the de la Vallée-Poussin theorem ([12] p. 174), $\left\{D^{\alpha} u_{n}\right\}$ is uniformly equi-integrable in $L^{1}(\Omega)$ for each $|\alpha| \leqq m$. On the other hand, as $\left\{D^{\alpha} u_{n}\right\}$ is bounded also in $L_{M}(\Omega)$ for each $|\alpha| \leqq m$, we can establish the existence of a subsequence of $\left\{u_{n}\right\}$ such that $D^{\alpha} u_{n} \rightarrow D^{\alpha} u$ in $L_{M}(\Omega)$ for the topology $\sigma\left(L_{M}(\Omega), E_{\bar{M}}(\Omega)\right)$ and also $D^{\alpha} u_{n} \rightarrow D^{\alpha} u$ in $L^{1}(\Omega)$ for each $|\alpha| \leqq m$. We can then show the desired result that the limit element $u \in W^{m} L_{M}(\Omega) \cap K$ is also in $V_{1}$ and solves (3.1) by verifying again the assertions (1) to (4). The proofs of these assertions remain true without any essential change. We mention the following two facts only. Firstly, (3.5) and (3.13) hold by Vitali's convergence theorem because of the equi-integrability of $\left\{D^{\alpha} u_{n}\right\}$. Secondly, $\left(f, u_{n}\right) \rightarrow(f, u)$ as $n \rightarrow \infty$, since $D^{\alpha} u_{n} \rightarrow D^{\alpha} u$ in $L_{M}(\Omega)$ for $\sigma\left(L_{M}(\Omega), E_{\bar{M}}(\Omega)\right)$ for all $|\alpha| \leqq m$ and $f=\left(f_{\alpha}\right) \in W^{-m} E_{\bar{M}}(\Omega)$.

For the boundary value problems subject to the boundary constraints which are associated to the subspace $V$ with $W_{0}^{m, 1}(\Omega) \subset V \subset W^{m, 1}(\Omega)$ the following generalisation to Theorem 2 can be derived from Theorem 3 .

Theorem 4. Let $\Omega$ be a bounded domain in $\boldsymbol{R}^{N}$ having the cone property and let the functions $g_{\alpha}$ satisfy the conditions $\left(\mathrm{H}_{1}\right),\left(\mathrm{H}_{2}\right)$ and $\left(\mathrm{H}_{3}\right)$. Then for each $f \in W^{-m} E_{\bar{M}}(\Omega)$ there exists $u$ in $V_{1} \cap W^{m} L_{M}(\Omega)$ such that (3.16) and (3.17) hold.

Remark 2. When $\|\cdot\|_{m, 1}$ and $|\cdot|_{m, 1}$ are equivalent norms in $V$ (for example $\left.V=W_{0}^{m, 1}(\Omega)\right)$, the coercivity condition (1.2) of $\left(\mathrm{H}_{1}\right)$ can be relaxed for the functions $g_{\alpha}$ with $|\alpha| \leqq m-1$. 


\section{Examples and applications}

In this section we shall indicate briefly some examples of the problems which can be solved by our existence theorems of the previous section. We start with mentioning some specific functions $g_{\alpha}$ which satisfy the conditions $\left(\mathrm{H}_{1}\right)$ or $\left(\mathrm{H}_{1}\right)^{*}$, $\left(\mathrm{H}_{2}\right)$ and $\left(\mathrm{H}_{3}\right)$.

1) $g_{\alpha}(t)=|t|^{p_{\alpha} \operatorname{sgn} t}$, where $0<p_{\alpha}<\infty$ for all $|\alpha| \leqq m$. The Sobolev space $W^{m, p}(\Omega)$ is determined by $p=\min \left\{p_{\alpha}+1:|\alpha| \leqq m\right\}$. In this case the partial differential operator $A$ defined by (1.1) has polynomial growth which may depend upon the direction. This kind of operator is called anisotropic in the literature (cf. [7] p. 191). Obviously the functions $g_{\alpha}$ satisfy the conditions $\left(\mathrm{H}_{1}\right)^{*},\left(\mathrm{H}_{2}\right)$ and $\left(\mathrm{H}_{3}\right)$.

2) $g_{\alpha}(t)=|t|^{p_{\alpha}} e^{|t|^{q_{\alpha}}} \operatorname{sgn} t$

where $0<p_{\alpha}, q_{\alpha}<\infty$ for all $|\alpha| \leqq m$. Then $W^{m, p}(\Omega)$ is defined as above and the operator $A$ is anisotropic with an exponential growth. The conditions $\left(\mathrm{H}_{1}\right)^{*},\left(\mathrm{H}_{2}\right)$ and $\left(\mathrm{H}_{3}\right)$ are satisfied.

3) $g_{\alpha}(t)=\left\{\begin{array}{l}t^{p_{\alpha}}, \text { if } \quad t \geqq 0, \\ -|t|^{q_{\alpha}} \log (1+|t|), \quad \text { if } t<0,\end{array}\right.$

where $0<p_{\alpha}, q_{\alpha}<\infty$ for all $|\alpha| \leqq m$. The conditions $\left(\mathrm{H}_{1}\right)^{*},\left(\mathrm{H}_{2}\right)$ and $\left(\mathrm{H}_{3}\right)$ are again satisfied. Note further that the operator $A$ is not odd (cf. [8], [10]).

4) As an example of functions $g_{\alpha}$ satisfying $\left(\mathrm{H}_{1}\right)$ but not $\left(\mathrm{H}_{1}\right)^{*}$ we mention

$$
g_{\alpha}(t)=\log (1+|t|) \operatorname{sgn} t \text { for all }|\alpha| \leqq m .
$$

The Sobolev-Orlicz space $W^{m} L_{M}(\Omega)$ is then defined by the function

$$
M(s)=\int_{0}^{s} g_{\alpha}(t) d t .
$$

The corresponding operator $A$ has slow growth, the coercivity condition (1.2) of $\left(\mathrm{H}_{1}\right)$ being still satisfied.

5) The functions $g_{\alpha}$ may, of course, be any combinations of the types mentioned above. A constant $c_{\alpha}$ can be added to each $g_{\alpha}$ with $|\alpha|=m$ and each $g_{\alpha}$ with $|\alpha| \leqq$ $m-1$ may be added by any bounded function $k_{\alpha}$.

We finally conclude with some examples of specific boundary value problems and convex sets $K$ appearing in the applications.

a) If $V=W_{0}^{m, p}(\Omega)$ with $1 \leqq p<\infty$, Theorem 2 or Theorem 4, respectively, establishes the existence of a weak solution $u$ for the Dirichlet problem

$$
\left\{\begin{array}{l}
\sum_{|\alpha| \leqq m}(-1)^{|\alpha|} D^{\alpha}\left(g_{\alpha}\left(D^{\alpha} u\right)\right)=f \text { in } \Omega, \\
D^{\alpha} u=0 \quad \text { on } \partial \Omega \text { for all }|\alpha| \leqq m-1
\end{array}\right.
$$


for each prescribed $f \in W^{-m, p^{\prime}}(\Omega)$ (resp. $f \in W^{-m} E_{\bar{M}}(\Omega)$ ), provided the functions $g_{\alpha}$ satisfy the conditions $\left(\mathrm{H}_{1}\right)^{*}$ (resp. $\left.\left(\mathrm{H}_{1}\right)\right),\left(\mathrm{H}_{2}\right)$ and $\left(\mathrm{H}_{3}\right)$.

Similarly, if $V=W^{m, p}(\Omega)$, the existence of a weak solution for the boundary value problem with Neumann type boundary constraints follows.

b) Let $\psi \in W^{1, \infty}(\Omega)$ be a given function such that $\psi(x) \leqq 0$ on the boundary $\partial \Omega$. Let

$$
K=\left\{v \in W_{0}^{1, p}(\Omega): v(x) \geqq \psi(x) \text { a.e. in } \Omega\right\}
$$

and let the functions $g_{\alpha}$ satisfy the conditions $\left(\mathrm{H}_{1}\right)$ to $\left(\mathrm{H}_{3}\right)$. The "obstacle problem" asks for a solution $u$ in $K$ of the variational inequality

$$
\int_{\Omega} \sum_{|\alpha| \leqq 1} g_{\alpha}\left(D^{\alpha} u\right)\left(D^{\alpha} w-D^{\alpha} u\right) d x \geqq(f, w-u) \quad \text { for all } \quad w \in K \cap W,
$$

where $W=W^{2, s}(\Omega) \cap W_{0}^{1, p}(\Omega), s>\max \{N, p\}$, with $1<p<\infty$ if $\left(\mathrm{H}_{1}\right)^{*}$ is satisfied, $p=1$ if only $\left(\mathrm{H}_{1}\right)$ is met. When $\psi(x) \leqq 0$ in the whole set $\Omega, 0 \in K$ and the existence of a solution $u$ in $K \cap V_{1}$ follows immediately from Theorem 1 for each $f \in W^{-1, p}(\Omega)$ and from Theorem 3 for each $f \in W^{-1} E_{\bar{M}}(\Omega)$, respectively. If $0 \notin K$, we may perform a change of variable $u \rightarrow \hat{u}=u-\psi^{+}$, where $\psi^{+}=\max \{\psi, 0\}$.

Then we set

$$
\hat{K}=\left\{\hat{u} \in W_{0}^{1, p}(\Omega): \hat{u}(x) \geqq \psi(x)-\psi^{+}(x) \text { in } \Omega\right\},
$$

whence $K$ contains the origin. The problem (4.2) is now equivalent to finding $\hat{u} \in \hat{K}$ such that

$$
\int_{\Omega} \sum_{|\alpha| \leqq 1} g_{x}\left(D^{\alpha} \hat{u}+D^{\alpha} \psi^{+}\right)\left(D^{\alpha} \hat{w}-D^{\alpha} \hat{u}\right) d x \geqq(f, \hat{w}-\hat{u}) \quad \text { for all } \hat{w} \in \hat{K} \cap W .
$$

Setting $\hat{g}_{\alpha}(x, t)=g_{\alpha}\left(t+D^{\alpha} \psi^{+}(x)\right)$ and formulating the analogous conditions for the functions of the type $g_{\alpha}(x, t)$ the existence theorems remain valid and the solvability of (4.3) follows.

c) Let $K=\left\{v \in W^{m, 1}(\Omega): v(x) \geqq 0\right.$ on $\left.\partial \Omega\right\}$. Then the variational inequality (3.1) is equivalent to a boundary value problem with certain boundary constraints (cf. [17] p. 99). When $K=\left\{v \in W_{0}^{1,1}(\Omega):|\nabla v(x)| \leqq 1\right.$ a.e. in $\left.\Omega\right\}$, (3.1) constitutes a problem of the type appearing in the theory of elasto-plasticity (cf. [17], p. 100).

\section{Uniqueness theorems}

Our purpose in this section is to deal with the uniqueness of solutions of the variational problems for the operator (1.1) discussed in Section 3. To this end we shall assume in what follows that all coefficients $g_{\alpha}$ are strictly increasing. For convenience we shall restrict ourselves to the second order operators and to the case where the strict form of the sign condition is fulfilled. Thus we can reformulate the conditions for the functions $g_{0}, g_{1}, \ldots, g_{N}$ as follows. 
$\left(\mathrm{E}_{1}\right)$ For each $i=0,1, \ldots, N$ the function $g_{i}: \boldsymbol{R} \rightarrow \boldsymbol{R}$ is continuous and strictly increasing, and satisfies $g_{i}(t) t \geqq 0$ for all $t \in \boldsymbol{R}$. There exists an Orlitz function $M$ and a constant $c_{1}>0$ such that for each $i=1,2, \ldots, N$,

$$
\begin{array}{lll}
g_{i}(t) t \geqq M(t)-c_{1} & \text { for all } & t \in \boldsymbol{R} . \\
g_{0}(t) t \geqq M(t)-c_{1} & \text { for all } & t \in \boldsymbol{R} .
\end{array}
$$

For each $i=0,1, \ldots, N$ we define the function $G_{i}$ by

$$
G_{i}(s)=\int_{0}^{s} g_{i}(t) d t
$$

which is convex obeying the inequality

$$
G_{i}(s)-G_{i}(t) \geqq g_{i}(t)(s-t) \text { for all } s, t \in \boldsymbol{R} .
$$

When $\Omega$ is a bounded subset of $\boldsymbol{R}^{N}$ having the cone property, $V$ is a closed subspace of $W^{1,1}(\Omega)$ containing $W_{0}^{1,1}(\Omega), \quad W=V \cap W^{2, s}(\Omega)$ with $s>N$, and $K$ is any closed convex subset of $V$ containing the origin, it follows from the conditions $\left(E_{1}\right)$ and $\left(E_{2}\right)$ and from Theorem 3 that the variational inequality

$$
\int_{\Omega} \sum_{i=0}^{N} g_{i}\left(D_{i} u\right)\left(D_{i} w-D_{i} u\right) d x \geqq(f, w-u) \text { for all } w \in K \cap W
$$

admits a solution $u$ in $K \cap V_{1} \cap W^{1} L_{M}(\Omega)$ for each given $f$ in $W^{-1} E_{\bar{M}}(\Omega)$, where

$$
V_{1}=\left\{v \in V: g_{i}\left(D_{i} v\right) D_{i} v \in L^{1}(\Omega) \text { and } g_{i}\left(D_{i} v\right) \in L^{1}(\Omega) \text { for } i=0,1, \ldots, N\right\} \text {. }
$$

On the other hand, by (5.2) each solution $u$ of (5.3) is also a solution of the inequality

$$
\int_{\Omega} \sum_{i=0}^{N}\left\{G_{i}\left(D_{i} w\right)-G_{i}\left(D_{i} u\right)\right\} d x \geqq(f, w-u) \text { for all } w \in K \cap W .
$$

Similarly, the solutions of the boundary value problem

$$
\int_{\Omega} \sum_{i=0}^{N} g_{i}\left(D_{i} u\right) D_{i} w d x=(f, w) \text { for all } w \in W
$$

with the property

$$
\int_{\Omega} \sum_{i=0}^{N} g_{i}\left(D_{i} u\right) D_{i} u d x \leqq(f, u)
$$

are also solutions of (5.4) with $K=V$. Our study on the uniqueness of solutions for (5.3) is based on the inequality (5.4) which, however, should hold true for all $w$ in $K \cap V_{2}$, where

$$
V_{2}=\left\{v \in V: G_{i}\left(D_{i} v\right) \in L^{1}(\Omega) \text { for all } i=0,1, \ldots, N\right\} .
$$

Note that $V_{1}$ is contained in $V_{2}$, since $G_{i}(t) \leqq g_{i}(t) t$ for all $t \in \boldsymbol{R}$ and $i=0,1, \ldots, N$. As $G_{i}(t) \geqq 0$ for all $t \in \boldsymbol{R},(5.4)$ is obviously satisfied for all $w \in V$ with $w \notin V_{2}$. The following simple lemma (cf. [3]) shows that the main problem in establishing 
the uniqueness for (5.3) or for the pair $\{(5.5),(5.6)\}$, respectively, is to extend (5.4) for all $w \in K \cap V_{2}$.

Lemma 5. Let the functions $g_{i}(i=0,1, \ldots, N)$ satisfy the conditions $\left(\mathrm{E}_{1}\right)$ and $\left(\mathrm{E}_{2}\right)$. Then the variational inequality

$$
\int_{\Omega} \sum_{i=0}^{N}\left\{G_{i}\left(D_{i} v\right)-G_{i}\left(D_{i} u\right)\right\} d x \geqq(f, v-u) \quad \text { for all } \quad v \in K \cap V_{2}
$$

admits at most one solution $u$ in $K \cap V_{1}$.

Proof. Let $u_{1}$ and $u_{2}$ be two solutions of (5.7) in $K \cap V_{1}$. Since $V_{1} \subset V_{2}$ and since each $G_{i}$ is convex, $(1 / 2)\left(u_{1}+u_{2}\right) \in V_{2}$. Thus we can set $v=(1 / 2)\left(u_{1}+u_{2}\right)$ in the inequalities

$$
\begin{gathered}
\int_{\Omega} \sum_{i=0}^{N}\left\{G_{i}\left(D_{i} v\right)-G_{i}\left(D_{i} u_{1}\right)\right\} d x \geqq\left(f, v-u_{1}\right), \\
\int_{\Omega} \sum_{i=0}^{N}\left\{G_{i}\left(D_{i} v\right)-G_{i}\left(D_{i} u_{2}\right)\right\} d x \geqq\left(f, v-u_{2}\right),
\end{gathered}
$$

and we obtain by addition,

$$
\int_{\Omega} \sum_{i=1}^{N}\left\{\frac{G_{i}\left(D_{i} u_{1}\right)-G_{i}\left(D_{i} u_{2}\right)}{2}-G_{i}\left(\frac{D_{i} u_{1}+D_{i} u_{2}}{2}\right)\right\} d x \leqq 0 .
$$

The conclusion $u_{1}=u_{2}$ follows now from the strict convexity of the functions $G_{i}$ for each $i=0,1, \ldots, N$.

A domain $\Omega$ in $\boldsymbol{R}^{N}$ is said to be starlike, if for any $r>1, \Omega_{\boldsymbol{r}}=\left\{x \in \boldsymbol{R}^{N}: r x \in \Omega\right\} \subset \subset \Omega$, i.e. $\bar{\Omega}_{r} \subset \Omega$ and $\bar{\Omega}_{r}$ is compact. We shall deal below with the starlike domains only. We are able to prove the uniqueness of solutions for the boundary value problems with the Dirichlet boundary conditions $\left(V=W_{0}^{1,1}(\Omega)\right)$ and with the Neumann type boundary conditions $\left(V=W^{1,1}(\Omega)\right.$ ), and for the obstacle problem (Example b, in Section 4). For simplicity, to avoid the discussion of various cases separately, we shall assume that $f$ is a given function in $L^{\infty}(\Omega)$. We start with

Theorem 5. Let $\Omega$ be a bounded starlike domain in $\boldsymbol{R}^{N}$ having the cone property and let the functions $g_{i}$ satisfy the condition $\left(\mathrm{E}_{1}\right)$. If $V=W_{0}^{1,1}(\Omega)$, then for each $f \in L^{\infty}(\Omega)$ there exists a unique $u$ in $V_{1}$ such that

$$
-\sum_{i=0}^{N} D_{i}\left(g_{i}\left(D_{i} u\right)\right)+g_{0}(u)=f \quad \text { in } \quad \Omega
$$

(in the sense of distributions), while (5.6) also holds.

Proof. Let $u$ be a solution of (5.8) satisfying (5.6). Such a solution exists by Theorem 4 and Remark 2. Thus

$$
\int_{\Omega} \sum_{i=0}^{N} g_{i}\left(D_{i} u\right) D_{i} w d x=(f, w) \text { for all } w \in C_{0}^{\infty}(\Omega)
$$


which, in view of (5.2) together with (5.6), implies that

$$
\int_{\Omega} \sum_{i=0}^{N}\left\{G_{i}\left(D_{i} w\right)-G_{i}\left(D_{i} u\right)\right\} d x \geqq(f, w-u) \text { for all } w \in C_{0}^{\infty}(\Omega) .
$$

To be able to apply Lemma 5 we must only show that (5.9) remains valid for all $w$ in $V_{2}$.

Let $v \in W_{0}^{1,1}(\Omega)$ with $G_{i}\left(D_{i} v\right) \in L^{1}(\Omega)$ for all $i=0,1, \ldots, N$. For any $\delta$ with $0<\delta<1$ we define $\Omega_{\delta}=\left\{x \in \boldsymbol{R}^{N}: \delta^{-1} x \in \Omega\right\}$, whence $\Omega_{\delta} \subset \subset \Omega$. Let further

$$
z_{\delta}(x)=\left\{\begin{array}{cll}
v\left(\delta^{-1} x\right), & \text { if } & x \in \Omega_{\delta} \\
0, & \text { if } & x \in \Omega \backslash \Omega_{\delta}
\end{array}\right.
$$

and $v_{\delta}(x)=\delta z_{\delta}(x)$ for all $x \in \Omega$. Then $z_{\delta}$ and $v_{\delta}$ belong to $W_{0}^{1,1}(\Omega)$ and $z_{\delta} \rightarrow v$ in $W_{0}^{1,1}(\Omega)$ as $\delta \rightarrow 1$ ([12] p. 70). Hence also $v_{\delta} \rightarrow v$ in $W_{0}^{1,1}(\Omega)$ as $\delta \rightarrow 1$. Furthermore, as

$$
\int_{\Omega} G_{0}(v(x)) d x=\delta^{-N} \int_{\Omega_{\delta}} G_{0}\left(v\left(\delta^{-1} x\right)\right) d x=\delta^{-N} \int_{\Omega} G_{0}\left(z_{\delta}(x)\right) d x,
$$

$G_{0}\left(z_{\delta}\right) \rightarrow G_{0}(v)$ and $\delta^{-N} G_{0}\left(z_{\delta}\right) \rightarrow G_{0}(v)$ in $L^{1}(\Omega)$ as $\delta \rightarrow 1$. Since $0 \leqq G_{0}(\delta s) \leqq G_{0}(s)$ for all $s \in \boldsymbol{R}$ and $0<\delta<1, G_{0}\left(v_{\delta}(x)\right) \leqq G_{0}\left(z_{\delta}(x)\right)$ for all $x \in \Omega$. On the other hand, $G_{0}\left(v_{\delta}(x)\right) \rightarrow G_{0}(v(x))$ a.e. in $\Omega$. Hence it follows by the dominated convergence theorem that $G_{0}\left(v_{\delta}\right) \rightarrow G_{0}(v)$ in $L^{1}(\Omega)$ as $\delta \rightarrow 1$. Similarly one can show that $G_{i}\left(D_{i} v_{\delta}\right) \rightarrow G_{i}\left(D_{i} v\right)$ in $L^{1}(\Omega)$ for each $i=1,2, \ldots, N$.

The next step is to approximate each $v_{\delta}$ by test functions. We denote

$$
v_{\delta \varepsilon}(x)=\int_{\Omega} j_{\varepsilon}(x-y) v_{\delta}(y) d y,
$$

the mollification of $v_{\delta}$ for any $\varepsilon>0$ ([1] p. 29). For each given $\delta, v_{\delta_{\varepsilon}} \in C_{0}^{\infty}(\Omega)$ and $v_{\delta \varepsilon} \rightarrow v_{\delta}$ in $W_{0}^{1,1}(\Omega)$ as $\varepsilon \rightarrow 0$ ([1] p. 52). We must show that $G_{i}\left(D_{i} v_{\delta \varepsilon}\right) \rightarrow G_{i}\left(D_{i} v_{\delta}\right)$ in $L^{1}(\Omega)$ for all $i=0,1, \ldots, N$. Indeed, by Jensen's inequality (see [12] p. 123),

$$
\begin{aligned}
& 0 \leqq G_{0}\left(v_{\delta \varepsilon}(x)\right)=G_{0}\left(\int_{\Omega} j_{\varepsilon}(x-y) v_{\delta}(y) d y\right) \\
& \leqq \int_{\Omega} j_{\varepsilon}(x-y) G_{0}\left(v_{\delta}(y)\right) d y=\left(G_{0}\left(v_{\delta}\right)\right)_{\varepsilon}(x),
\end{aligned}
$$

where $\left(G_{0}\left(v_{\delta}\right)\right)_{\varepsilon} \rightarrow G_{0}\left(v_{\delta}\right)$ in $L^{1}(\Omega)$ ([1] p. 30). Therefore $G_{0}\left(v_{\delta \varepsilon}\right) \rightarrow G_{0}\left(v_{\delta}\right)$ in $L^{1}(\Omega)$ again by the dominated convergence theorem. For each $i=1,2, \ldots, N$ we similarly have

$$
G_{i}\left(D_{i} v_{\delta \varepsilon}(x)\right)=G_{i}\left(\left(D_{i} v_{\delta}\right)_{\varepsilon}(x)\right) \leqq\left(G_{i}\left(D_{i} v_{\delta}\right)_{\varepsilon}\right)(x),
$$

and the convergence $G_{i}\left(D_{i} v_{\delta \varepsilon}\right) \rightarrow G_{i}\left(D_{i} v_{\delta}\right)$ in $L^{1}(\Omega)$ can be derived accordingly.

The final step of the proof is to set $w=v_{\delta \varepsilon}$ in (5.9), to send firstly $\varepsilon \rightarrow 0$ and then $\delta \rightarrow 1$ to obtain the assertion.

For the Neumann type boundary value problems some modifications are necessary. 
Theorem 6. Let $\Omega$ be a bounded starlike domain in $\boldsymbol{R}^{N}$ having the cone property and let the functions $g_{i}$ satisfy the conditions $\left(\mathrm{E}_{1}\right)$ and $\left(\mathrm{E}_{2}\right)$. Let $V=W^{1,1}(\Omega)$ and $W=V \cap W^{2, s}(\Omega)$ with $s>N$. Then for each $f \in L^{\infty}(\Omega)$ there exists a unique $u$ in $V_{1} \cap W^{1} L_{M}(\Omega)$ such that (5.5) and (5.6) hold.

Proof. Let $u$ be a solution in $V_{1} \cap W^{1} L_{M}(\Omega)$ of the pair $\{(5.5),(5.6)\}$. A solution exists by Theorem 4 . As above in the proof of Theorem 5 we can conclude that

$$
\int_{\Omega} \sum_{i=0}^{N}\left\{G_{i}\left(D_{i} w\right)-G_{i}\left(D_{i} u\right)\right\} d x \geqq(f, w-u) \quad \text { for all } \quad w \in W^{2, s}(\Omega) .
$$

In view of Lemma 5 it will be sufficient to find, for each $v \in W^{1,1}(\Omega)$ with $G_{i}\left(D_{i} v\right) \in L^{1}(\Omega)$ for all $i=0,1, \ldots, N$, a suitable approximation in $W^{2, s}(\Omega)$. For any $\delta>1$ we define $\Omega_{\delta}=\left\{x \in \boldsymbol{R}^{N}: \delta^{-1} x \in \Omega\right\}$ so that $\Omega \subset \subset \Omega_{\delta}$. Let $z_{\delta}(x)=v\left(\delta^{-1} x\right)$ for all $x \in \Omega_{\delta}$. Then $z_{\delta} \rightarrow v$ in $W^{1,1}(\Omega)$ as $\delta \rightarrow 1$ and, furthermore,

$$
\int_{\Omega} G_{0}(v(x)) d x=\delta^{-N} \int_{\Omega_{\delta}} G_{0}\left(v\left(\delta^{-1} x\right)\right) d x \geqq \delta^{-N} \int_{\Omega} G_{0}\left(z_{\delta}(x)\right) d x,
$$

implying that $G_{0}\left(z_{\delta}\right) \in L^{1}(\Omega)$ and $G_{0}\left(z_{\delta}\right) \rightarrow G_{0}(v)$ in $L^{1}(\Omega)$ as $\delta \rightarrow 1$. For each $i=1,2, \ldots, N$, we have further $D_{i} z_{\delta}(x)=\delta^{-1} D_{i} v\left(\delta^{-1} x\right)$ and hence

$$
\begin{gathered}
\int_{\Omega} G_{i}\left(D_{i} v(x)\right) d x=\delta^{-N} \int_{\Omega_{\delta}} G_{i}\left(D_{i} v\left(\delta^{-1} x\right)\right) d x \geqq \delta^{-N} \int_{\Omega} G_{i}\left(D_{i} v\left(\delta^{-1} x\right)\right) d x \\
\geqq \delta^{-N} \int_{\Omega} G_{i}\left(\delta^{-1} D_{i} v\left(\delta^{-1} x\right)\right) d x=\delta^{-N} \int_{\Omega} G_{i}\left(D_{i} z_{\delta}(x)\right) d x .
\end{gathered}
$$

Consequently, $G_{i}\left(D_{i} z_{\delta}\right) \rightarrow G_{i}\left(D_{i} v\right)$ in $L^{1}(\Omega)$ for each $i=1,2, \ldots, N$ follows by a previous argument.

Each $z_{\delta}$ can now be approximated by the mollification $z_{\delta \varepsilon}$ such that $z_{\delta \varepsilon} \rightarrow z_{\delta}$ in $W^{1,1}(\Omega)$ as $\varepsilon \rightarrow 0$, because $\Omega \subset \subset \Omega_{\delta}$ (see [1] p. 52). It is also obvious that $z_{\delta \varepsilon} \in W^{2, s}(\Omega)$ for any $\varepsilon>0$ and $\delta>1$. We can complete the proof as the proof of Theorem 5 by showing that $G_{i}\left(D_{i} z_{\delta \varepsilon}\right) \rightarrow G_{i}\left(D_{i} z_{\delta}\right)$ in $L^{1}(\Omega)$ for all $i=0,1, \ldots, N$ and letting then $\varepsilon \rightarrow 0$ and finally $\delta \rightarrow 1$ in (5.10) with $w=z_{\delta \varepsilon}$.

We shall close our discussion with the following uniqueness theorem for the obstacle problem.

Theorem 7. Let $\Omega$ be a bounded starlike domain in $\boldsymbol{R}^{N}$ having the cone property, let the functions $g_{i}$ satisfy the condition $\left(\mathrm{E}_{1}\right)$, let $K=\left\{v \in W_{0}^{1,1}(\Omega): v(x) \geqq \psi(x)\right.$ a.e. in $\left.\Omega\right\}$, where $\psi$ is a given function in $W^{2, \infty}(\Omega)$ with $\psi \leqq 0$ in $\Omega$, and let $V=W_{0}^{1,1}(\Omega)$, $W=V \cap W^{2, s}(\Omega)$ with $s>N$. Then for each $f \in L^{\infty}(\Omega)$ there exists a unique $u$ in $K \cap V_{1}$ such that

$$
\int_{\Omega} \sum_{i=0}^{N} g_{i}\left(D_{i} u\right)\left(D_{i} w-D_{i} u\right) d x \geqq(f, w-u) \quad \text { for all } \quad w \in K \cap W .
$$


Proof. By Theorem 3 and Remark 2 a solution $u \in K \cap V_{1}$ of (5.11) exists. By the previous argument we get from (5.11) the inequality

$$
\int_{\Omega} \sum_{i=0}^{N}\left\{G_{i}\left(D_{i} w\right)-G_{i}\left(D_{i} u\right)\right\} d x \geqq(f, w-u) \text { for all } w \in K \cap W .
$$

For a given $v \in K$ with $G_{i}\left(D_{i} v\right) \in L^{1}(\Omega)$ for all $i=0,1, \ldots, N$, we define $v_{\delta}$ and $v_{\delta \varepsilon}$ with $0<\delta<1$ and $\varepsilon>0$ as in the proof of Theorem 5. Since these approximations are not necessarily in $K$, we also consider a map $w \rightarrow w_{\psi}$ in $W^{1,1}(\Omega)$ defined by

$$
w_{\psi}(x)=\max \{w(x), \psi(x)\}=[w-\psi]^{+}(x)+\psi(x) .
$$

As $\psi(x) \leqq 0$ a.e. in $\Omega, 0_{\psi}=0$. Moreover, it is known that

$$
D_{i} w_{\psi}(x)=\left\{\begin{array}{lll}
D_{i} w(x), & \text { if } & w(x)>\psi(x), \\
D_{i} \psi(x), & \text { if } & w(x) \leqq \psi(x)
\end{array}\right.
$$

and that the map $w \rightarrow w_{\psi}$ is continuous in $W^{1,1}(\Omega)$. Then it is also clear that $v_{\psi}=v$, $\left(v_{\delta}\right)_{\psi} \in K$ and $\left(v_{\delta \varepsilon}\right)_{\psi} \in K$. Since $v_{\delta \varepsilon} \rightarrow v_{\delta}$ and $v_{\delta} \rightarrow v$ in $W^{1,1}(\Omega)$ as $\varepsilon \rightarrow 0$ and $\delta \rightarrow 1$, respectively, $\quad\left(v_{\delta_{\varepsilon}}\right)_{\psi} \rightarrow\left(v_{\delta}\right)_{\psi}$ and $\left(v_{\delta}\right)_{\psi} \rightarrow v_{\psi}=v$ in $W^{1,1}(\Omega)$. Next we show that $G_{i}\left(D_{i}\left(\left(v_{\delta_{\varepsilon}}\right)_{\psi}\right)\right) \rightarrow G_{i}\left(D_{i} v\right)$ in $L^{1}(\Omega)$ for each $i=0,1, \ldots, N$. Indeed, it is readily seen that $D_{i}\left(\left(v_{\delta \varepsilon}\right)_{\psi}\right)(x) \rightarrow D_{i} v(x)$ a.e. in $\Omega$ (for a subsequence). By continuity also $G_{i}\left(D_{i}\left(\left(v_{\delta \varepsilon}\right)_{\psi}\right)(x)\right) \rightarrow G_{i}\left(D_{i} v(x)\right)$ a.e. in $\Omega$. From the proof of Theorem 5 we already know that $G_{i}\left(D_{i} v_{\delta_{\varepsilon}}\right) \rightarrow G_{i}\left(D_{i} v\right)$ in $L^{1}(\Omega)$. Hence there exist functions $h_{0}, h_{1}, \ldots, h_{N}$ in $L^{1}(\Omega)$ such that $G_{i}\left(D_{i} v_{\delta \varepsilon}(x)\right) \leqq h_{i}(x)$ a.e. in $\Omega$. Consequently,

$$
\begin{aligned}
G_{i}\left(D_{i}\left(\left(v_{\delta \varepsilon}\right)_{\psi}\right)(x)\right) & \leqq G_{i}\left(D_{i} v_{\delta \varepsilon}(x)\right)+G_{i}\left(D_{i} \psi(x)\right) \\
& \leqq h_{i}(x)+G_{i}\left(D_{i} \psi(x)\right),
\end{aligned}
$$

where $h_{i}+G_{i}\left(D_{i} \psi\right) \in L^{1}(\Omega)$ for all $i=0,1, \ldots, N$. Hence $G_{i}\left(D_{i}\left(\left(v_{\delta \varepsilon}\right)_{\psi}\right)\right) \rightarrow G_{i}\left(D_{i} v\right)$ in $L^{1}(\Omega)$, by the dominated convergence theorem.

Because $\left(v_{\delta \varepsilon}\right)_{\psi}$ is not necessarily in $W^{2, s}(\Omega)$ and therefore not in $W$, the final step of the proof is to find for each fixed $\left(v_{\delta \varepsilon}\right)_{\psi}$ an approximation in $W$. Indeed, $1^{\text {et }} w=v_{\delta \varepsilon} \in C_{0}^{\infty}(\Omega)$ be given. For any $n \in N$ we define a function $\varphi_{n}: R \rightarrow R$ by

$$
\varphi_{n}(t)=\left\{\begin{array}{l}
0, \quad \text { if } t \leqq \frac{1}{2 n}, \\
n t^{2}-\frac{1}{4 n}-t, \quad \text { if } \quad \frac{1}{2 n}<t \leqq \frac{1}{n}, \\
t-\frac{3}{4 n}, \quad \text { if } \quad \frac{1}{n}<t .
\end{array}\right.
$$

The mollification of $\varphi_{n}$ is denoted by $\varphi_{n \sigma}$, where $0<\sigma<1 / 4 n$. Then $\varphi_{n \sigma} \in C^{\infty}(R)$, $\varphi_{n \sigma} \geqq 0$ and $\varphi_{n \sigma}=0$ for all $t \leqq 1 / 4 n$. Moreover, there exists a function $\varrho \in C_{0}^{\infty}(\Omega)$ such that $0 \leqq \varrho(x) \leqq 1$ in $\Omega$ and $\varrho(x)=1$ in the support of $w$. Now we can set 
$w_{n \sigma}=\left\{\varphi_{n \sigma}(w-\psi)+\psi\right\} \varrho$. Hence it is obvious that $w_{n \sigma} \in W_{0}^{1,1}(\Omega) \cap W^{2, \infty}(\Omega), w_{n \sigma} \geqq$ $\psi \varrho \geqq \psi$, i.e. $w_{n \sigma}$ is also in $K$. As $\varphi_{n}$ and $\varphi_{n}^{\prime}$ are continuous and $w-\psi$ is bounded in $W^{1, \infty}(\Omega)$, we can easily conclude that $w_{n \sigma} \rightarrow w_{\psi} \varrho=w_{\psi}$ in $W^{1,1}(\Omega)$ as $\sigma \rightarrow 0$ and $n \rightarrow \infty$, while $\varrho(x)=1$ in $\operatorname{supp}\left(w_{\psi}\right)$. Since $\left|\varphi_{n \sigma}(t)\right| \leqq|t|+1$ and $\left|\varphi_{n \sigma}^{\prime}(t)\right| \leqq 1$ for all $t$, we can apply the dominated convergence theorem to conclude that $G_{0}\left(w_{n \sigma}\right) \rightarrow$ $G_{0}\left(w_{\psi}\right)$ and $G_{i}\left(D_{i} w_{n \sigma}\right) \rightarrow G_{i}\left(D_{i} w_{\psi}\right)$ for all $i=1,2, \ldots, N$ in $L^{1}(\Omega)$. Our proof is complete if we substitute $w_{n \sigma}$ with $w=v_{\delta \varepsilon}$ into (5.12) and let $\sigma \rightarrow 0, n \rightarrow \infty, \varepsilon \rightarrow 0$ and finally $\delta \rightarrow 1$.

\section{References}

[1] Adams, R. A.: Sobolev spaces. - Academic Press, New York-London, 1975.

[2] Brezis, H.: Équations et inéquations non linéaires dans les espaces vectoríels en dualité. Ann. Inst. Fourier (Grenoble) 18, 1968, 115-175.

[3] Brezis, H., and F. E. Browder: Strongly nonlinear elliptic boundary value problems. - Ann. Scuola Norm. Pisa Cl. Sci (4) 5, 1978, 587-603.

[4] Browder, F. E.: Existence theorems for nonlinear partial differential equations. - Global Analysis (Proc. Sympos. Pure Math. Vol. XVI, Berkeley, Calif. 1968), Amer. Math. Soc., Providence, R. I., 1970, 1-60.

[5] Browder, F. E.: Existence theory for boundary value problems for quasilinear elliptic systems with strongly nonlinear lower order terms. - Partial differential equations (Proc. Sympos. Pure Math. Vol. XXIII, Berkeley, Calif., 1971), Amer. Math. Soc., Prov* idence, R. I., 1973, 269-286.

[6] Donaldson, T.: Nonlinear elliptic boundary value problems in Orlicz-Sobolev spaces. - J. Differential Equations 10, 1971, 507-528.

[7] Gossez, J. P.: Nonlinear elliptic boundary value problems for equations with rapidly (or slowly) increasing coefficients. - Trans. Amer. Math. Soc. 190, 1974, 163-205.

[8] Gossez, J. P.: Un problème de Dirichlet fortement non linéaire. - Boll. Un. Mat. Ital. A (5) $14,1977,118-125$.

[9] Gossez, J. P.: A remark on strongly nonlinear elliptic boundary value problems. - Bol. Soc. Brasil. Mat. 8, 1977, 53-63.

[10] Gossez, J. P., and P. Hess: Sur certains problèmes aux limites elliptiques fortement non linéaires. - C. R. Acad. Sci. Paris Sér. A 278, 1974, 343-345.

[11] HESS, P.: Variational inequalities for strongly nonlinear elliptic operators. - J. Math. Pures Appl. (9) 52, 1973, 285-297.

[12] Kufner, A., John, O., and S. Fučík: Function spaces. - Noordhoff International Publishing, Leyden; Academia, Praque, 1977.

[13] LANDES, R.: Quasilinear elliptic operators and weak solutions of the Euler equation. - Manuscripta Math. 27, 1979, 47-72.

[14] LANDES, R.: On Galerkin's method in the existence theory of quasilinear elliptic equations. - J. Funct. Anal. (to appear).

[15] LeRAY, J., and J. L. Lions: Quelques résultats de Višik sur les problèmes elliptiques non linéaires par les méthodes de Minty_Browder. - Bull. Soc. Math. France 93, 1965, 97-107.

[16] Lions, J. L.: Quelques méthodes de résolution des problèmes aux limites non linéaires. Dunod, Gauthier-Villars, Paris, 1969.

[17] Lions, J. L.: Partial differential inequalities. - Russian Mathematical Surveys, 27:2, 1972, 91-159. 
[18] Mustonen, V.: Strongly nonlinear elliptic variational inequalities in unbounded domains. Ann. Acad. Sci. Fenn. Ser. A. I. Math. 3, 1977, 59-74.

[19] Mustonen, V.: A class of strongly nonlinear variational inequalities in unbounded domains. J. London Math. Soc. (2) 19, 1979, 319-328.

[20] Simader, C. G.: Über schwache Lösungen des Dirichlet problems für streng nichtlineare elliptische Differentialgleichungen. - Math. Z. 150, 1976, 1-26.

[21] WebB, J. R. L.: Strongly nonlinear elliptic equations. - Journées d'Analyse Non Linéaire (Proc. Conf., Besançon, 1977), Lecture Notes in Mathematics 665, Springer-Verlag, Berlin-Heidelberg-New York, 1978, 242-256.

[22] Weвв, J. R. L.: Boundary value problems for strongly nonlinear elliptic equations. - J. London Math. Soc. (2) 21, 1980, 123-132.

University of Oulu

Department of Mathematics

SF-90570 Oulu 57

Finland
Universität Bayreuth

Mathematisches Institut

Postfach 3008

D 8580 Bayreuth

BRD

Received 6 March 1981 